\title{
Reply: Isolated tumour cells and micrometastases in intraductal breast cancer: a simple mechanical question in some cases
}

\author{
T Osako*,1,2, T Iwase $^{3}$ and F Akiyama ${ }^{1}$ \\ ${ }^{1}$ Division of Pathology, The Cancer Institute of the Japanese Foundation for Cancer Research, 3-8-31, Ariake, Koto-ku, \\ Tokyo 135-8550, Japan; 'Department of Pathology, The Cancer Institute Hospital of the Japanese Foundation for Cancer \\ Research, 3-8-31, Ariake, Koto-ku, Tokyo 135-8550, Japan and ${ }^{3}$ Breast Oncology Center, The Cancer Institute Hospital of the \\ Japanese Foundation for Cancer Research, 3-8-31, Ariake, Koto-ku, Tokyo 135-8550, Japan
}

Sir,

We appreciate the data and comments provided by PiñeroMadrona et al (2013) regarding our recent publication (Osako et al, 2012). In our study, we showed that the number of patients with a postoperative diagnosis of ductal carcinoma in situ (DCIS; intraductal breast cancer) and sentinel lymph node (SN) metastasis increased significantly after the introduction of molecular wholenode analysis using the one-step nucleic acid amplification (OSNA) assay for SN biopsies. Piñero-Madrona et al (2013) also presented a case series of DCIS with SN metastasis detected using the OSNA assay. Their study gave similar results to ours as they showed that the OSNA assay was able to detect SN micrometastasis in DCIS and that there were $<1500$ copies of cytokeratin 19 mRNA per microlitre.

By definition, DCIS does not metastasise to the lymph nodes because the tumour is limited to the epithelial layer and does not reach lymphatic vessels. Therefore, as a possible explanation for the pathogenesis of nodal metastases in DCIS, we propose that true metastases arise from the occult invasion of the primary tumour and iatrogenic dissemination of benign epithelial or tumour cells during preoperative breast biopsy (Osako et al, 2012). On the basis of their findings, Piñero-Madrona et al (2013) also advocate the theory of iatrogenic dissemination resulting from preoperative needle biopsy.

Our more recent study also suggests that true metastasis from occult invasion may be a potent pathogenic mechanism (Osako et al, 2013). In this study, we compared the detection rates of occult invasion between SN-positive and SN-negative DCIS through exhaustive step sectioning of the primary tumour blocks. The subjects comprised 24 patients selected from among 285 patients who had received a routine postoperative diagnosis of DCIS. This is the same population as the OSNA cohort of the 2012 study (Osako et al, 2012). Of these 24 patients, 12 were SN positive and 12 were SN negative, and the latter were selected from among $273 \mathrm{SN}$-negative patients with characteristics similar to those of the control group. We analysed 1,830 step-sectioned slides and found that the incidence of occult invasion in the $\mathrm{SN}$-positive group was almost double that in the SN-negative group (7/12 (58.3\%) vs 3/12 (25.0\%)).

Despite our success, we were still unable to detect occult invasion in $\sim 40 \%$ of patients with DCIS and SN metastasis. Although this study has some limitations that may have prevented its detection, we believe that the iatrogenic dissemination theory remains one of the most likely explanations for the pathogenesis of nodal metastasis. It seems probable, therefore, that nodal metastasis in postoperatively diagnosed DCIS actually involves both true metastases (pT1mi/1a-pN1mi-M0 tumours) and iatrogenic dissemination (pTis-pN0-M0 tumours). However, the histopathological examination of primary tumours and lymph nodes and the OSNA assay for lymph nodes have only a limited ability to distinguish between true metastasis and iatrogenic dissemination. Novel markers for tumour cells in the node are still required to help determine which of these possibilities is correct, and follow-up of patients for whom DCIS and SN metastasis was detected using the OSNA assay is still needed to elucidate the prognostic significance of nodal metastases.

\section{REFERENCES}

Osako T, Iwase T, Kimura K, Horii R, Akiyama F (2013) Detection of occult invasion in ductal carcinoma in situ of the breast with sentinel node metastasis. Cancer Sci doi:10.1111/cas.12095.

Osako T, Iwase T, Kimura K, Masumura K, Horii R, Akiyama F (2012) Incidence and possible pathogenesis of sentinel node micrometastases in ductal carcinoma in situ of the breast detected using molecular whole lymph node assay. Br J Cancer 106(10): 1675-1681.

Piñero-Madrona P, Ruiz- Maciá JA, Zaragoza-Zaragoza C, Polo-García L, Sola-Pérez J (2013) Isolated tumour cells and micrometastases in intraductal breast cancer: a simple mechanical question in some cases. Br J Cancer 108: 1919-1920. 